\title{
Evaluation of the Relationship Between Serum Sodium Concentration and Mortality Rate in ICU Patients with Traumatic Brain Injury
}

\author{
Babak Ali Kiaei, ${ }^{1}$ Darioush Moradi Farsani, ${ }^{1,}{ }^{*}$ Keyvan Ghadimi, ${ }^{2}$ and Mahnaz Shahali ${ }^{2}$ \\ ${ }^{1}$ Assistant Professor, Department of Anesthesiology, Faculty of Medicine, Isfahan University of Medical Sciences, Isfahan, IR Iran \\ ${ }^{2}$ School of Medicine, Isfahan University of Medical Sciences, Isfahan, IR Iran \\ "Corresponding author: Darioush Moradi Farsani, Assistant Professor, Department of Anesthesiology, Faculty of Medicine, Isfahan University of Medical Sciences, Isfahan, IR \\ Iran. Tel: +98-9133050830, E-mail: dmoradi@med.mui.ac.ir
}

Received 2018 February 23; Revised 2018 April 13; Accepted 2018 May 09.

\begin{abstract}
Background: Traumatic brain injury (TBI) is a major cause of death and disability globally, and serum sodium disorders are the most common and probably the most poorly understood electrolyte disorders in neurological diseases.

objectives: The aim of this study was to evaluate the serum sodium level and its disorders including hyponatremia and hypernatremia in patients with TBI and evaluate the sodium levels at the time of admission, the first week, and the second week after admission.

Methods: This observational prospective descriptive-analytic study included patients with TBI admitted to the intensive care unit (ICU) of Saint Al-Zahra hospital, Isfahan, Iran during 2015 - 2016. Patients' demographic factors, hemodynamic and paraclinical data were documented in the information forms and imported to and analyzed using SPSS v.22. All of the statistical tests were conducted at an error level of $5 \%$.

Results: Demographic indices were not significantly different in the first and second weeks of the study between patients who died and the recovered or survived individuals. It was found that the mean of serum sodium was $146.26 \mathrm{mEq} / \mathrm{L}$ in patients who died and 142.66 in alive patients $(\mathrm{P}<0.001)$ in the first week and 147.8 in the patients who died and 144.03 in alive patients $(\mathrm{P}<0.001)$ in the second week.

Conclusions: The mortality prevalence of patients with TBI admitted to the ICU who have dysnatremia is high and can increase the risk of mortality remarkably. The mortality risk of hypernatremia is significantly higher than that of hyponatremia.
\end{abstract}

Keywords: Intensive Care Unit (ICU), Mortality, Serum Sodium, Traumatic Brain Injury (TBI)

\section{Background}

Traumatic brain injury or TBI is a major cause of death and disability globally. Sodium disturbances are common in patients with brain injury because of the major role that the central nervous system plays in the regulation of sodium and water homeostasis. In addition, treatment of the injured brain can itself disturb the regulation of sodium. In addition, serum sodium disorders are the most common and probably the most poorly understood electrolyte disorders in neurological diseases (1-3). Although TBI has been recognized as an untreatable term and predicts the mortality, the progress in trauma care systems, including pre-hospital care and intensive care, improves the survival of traumatic patients (4). Patients with severe cerebral trauma have a high risk of developing dysnatremia over the course of admission to the intensive care unit (ICU), which is due to predisposing conditions such as sensory disturbances, changes in thirst, central diabetes insipidus alongside polyuria, and increased imperceptible water loss (5). In addition, these patients often receive mannitol and hypertonic saline solution for the purpose of reducing cerebral edema and controlling intracranial pressure (6). The occurrence of brain death is accompanied by the occurrence of subsequent events in the body. Hemodynamic and electrolytic disorders of hemostasis, as well as various hormonal disturbances, may occur in these patients (7). Therefore, the awareness of these disorders in order to prevent and resolve them with the aim of the patient's survival is of special importance. Considering that disorders of hemodynamic, coagulation, electrolyte (especially Na disturbances), blood sugar, and hormonal systems can cause a large percentage of patients with brain death, it is necessary to identify these disorders to prevent and treat them (8). 
Serum sodium concentration in humans is typically between 135 and $145 \mathrm{mEq} / \mathrm{L}$. Hyponatremia with a sodium concentration of less than $135 \mathrm{mEq} / \mathrm{L}$ means an increase in the ratio of total water to sodium and is seen in various medical conditions such as congestive heart failure, liver disease, and syndrome of inappropriate antidiuretic hormone secretion. Hyponatremia is the most common electrolyte disorder in hospitalized patients, and in some cases, up to $30 \%$ of patients are involved. The risk of death during the hospitalization period is increased by $50 \%$ in patients with hyponatremia compared to patients with normal serum levels $(5,6)$. On the other hand, sodium increase can also be due to dehydration, congestive heart failure, Cushing's disease, liver failure, high sodium diet, osmotic diuresis (secondary to hyperglycemia), azotemia, medications (such as contrast agents, mannitol, etc.), or central diabetes insipidus (DI) or nephrogenic DI (2). As a water balance disorder, Hypernatremia is seen in 6 - $9 \%$ of very ill patients and is associated with increased mortality risk (6).

Sodium disorder occurs by the onset of brain death in many patients. Brain death is one of the most important complications coming after TBI, which is associated with many changes in hemodynamics, homeostasis, and other disorders, and recognition of these disorders is important in identifying the brain death patients who are organ donor candidates. Therefore, the identification of markers that could predict the occurrence of brain death in patients in order to carry out therapeutic procedures and prevent the destruction of organs that can be donated seems indispensable. However, not many studies have been still conducted regarding this issue. This is while evaluating dysnatremia in patients with TBI and its relation to the occurrence of mortality is necessary.

\section{Objectives}

Therefore, the aim of this study was to evaluate dysnatremia and its relation to the occurrence of mortality in patients with TBI.

\section{Methods}

This is an observational prospective descriptiveanalytic study. All patients with moderate to severe TBI admitted to the ICU of Saint Al-Zahra hospital, Isfahan, Iran during 2015 - 2016 took part in this study. The sample size was calculated using the same formula for sample size estimation used in studies of prevalence and considering the confidence interval of 95\%; hypernatremia prevalence in TBI patients due to the lack of a similar study was considered 0.5 and acceptable error rate of 0.1 was used. Thus, the sample size was estimated to be 95 , and in order to achieve more reliability, 100 patients were studied.

The inclusion criteria consisted of patients with TBI admitted to the ICU of Saint Al-Zahra hospital. The informed consent of the patient's companions for participating in the study was acquired. Exclusion criteria consisted of patients with underlying renal diseases, patients admitted to the emergency unit for more than 48 hours, and patients companions not willing to participate in the study. In addition, patients that presented other potential causes of sodium disorders and systemic trauma (especially abdominal and orthopedic trauma because of that these lesions often involve blood loss and need resuscitation with saline solution) were excluded from the study. The hypertonic saline solution was not used in our cases. They did not receive diuretics and were free from renal or other endocrine diseases; in addition, patients with mild (Glasgow Coma Scale or GCS > 13) TBI were not considered in the study.

100 patients (Figure 1) who met the inclusion criteria were selected by the order of their entry into the ICU. According to the treatment protocol, blood parameters including daily sodium (sodium disorders were considered as disorders presenting serum sodium $<135 \mathrm{mEq} / \mathrm{L}$ or $>145$ $\mathrm{mEq} / \mathrm{L}$ ), potassium, calcium, magnesium, and phosphate, as well as coagulation factors including PT, PTT, and INR twice a week, daily blood acidity and the level of consciousness of patients with Glasgow score and Apache II scoring at the time of admission were measured according to the relevant protocol and documented in the information form. The mean level of these markers was measured at the end of the first and second weeks, and all patients were followed up as long as they were hospitalized in the ICU. The outcome for each patient, whether death or recovery, was determined and recorded in the patient's profile. The other characteristics of the patients analyzed were as follows: mean arterial blood pressure, mechanical ventilation time, and hospitalization period.

\subsection{Statistical Analysis}

Patient information including demographic factors and paraclinical findings was recorded in a checklist made by the study conductor and entered into SPSS v.22 software. Statistical analyses were conducted using descriptive and analytical statistics. In the descriptive section, the mean and standard deviation of paraclinical findings were presented as the main variables in different groups (dead and recovered) and all demographic and clinical characteristics of patients were reported based on descriptive crite- 


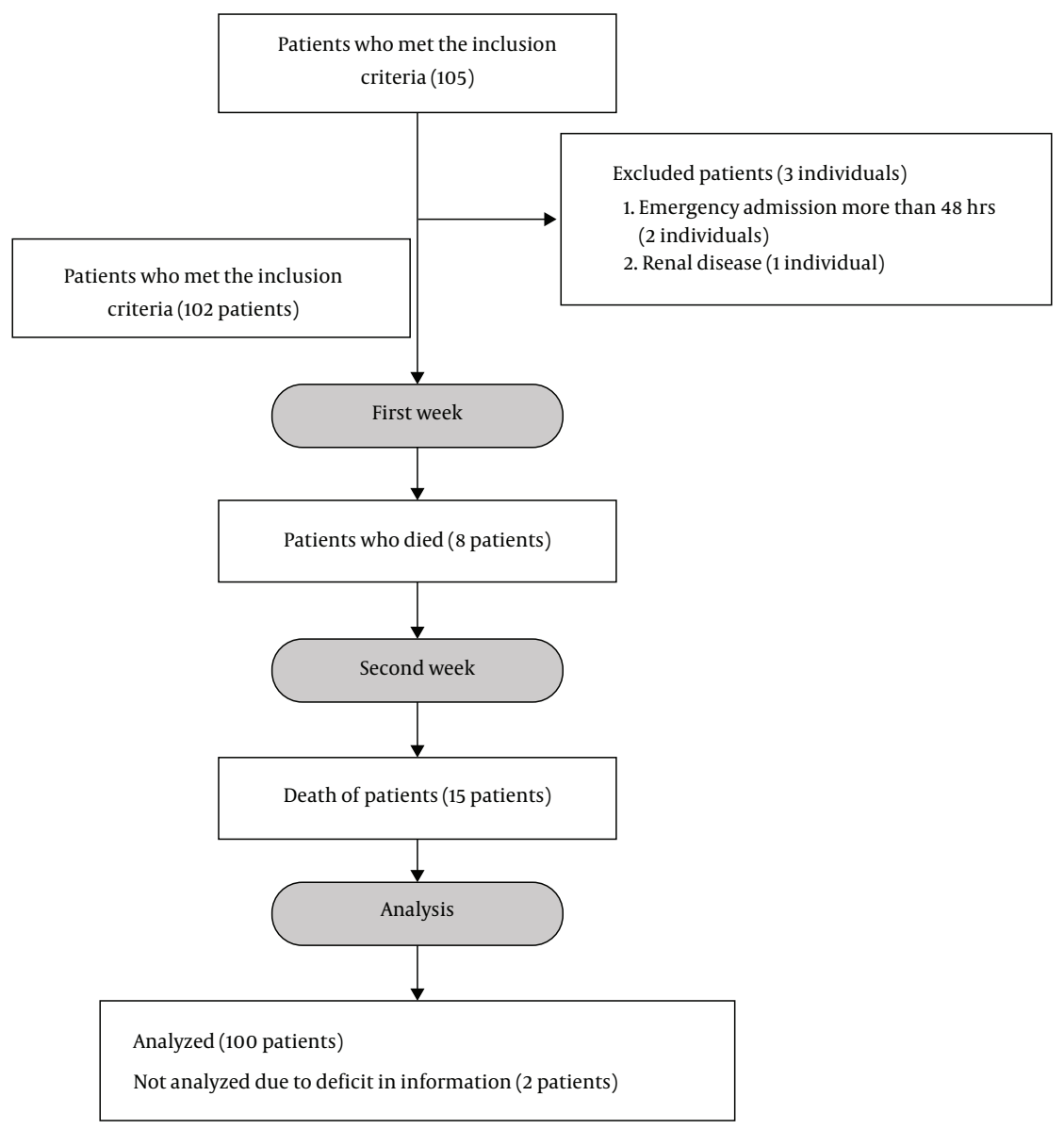

Figure 1. Study flowchart

ria. In the analytical section, based on the establishment of statistical presumptions, proportional parametric and nonparametric tests were used. Chi-square test was used to analyze the qualitative findings while to compare quantitative data, independent T-test and Mann-Whitney were used. To compare the changes in data in the first and second weeks, we used paired samples T-test. All of the tests were conducted at an error level of $5 \%$.

\section{Results}

Of the 100 patients enrolled in this study, 52 (52\%) were male and 48 (48\%) were female. The mean age of the subjects was $69.1 \pm 10.64$ years (38 - 80 years). Out of 100 patients, 23 (23\%) died within two weeks. Our results showed that demographic indices such as age $(P=0.321)$ and sex $(P=0.985$ ), electrolytes (calcium, potassium, phosphorus, and magnesium $)(P>0.05)$, hemodynamic changes (mean blood pressure and heart rate $)(\mathrm{P}>0.05)$, coagulation disorders (PT, PTT, and INR) $(\mathrm{P}>0.05)$, mechanical ventilation time $(\mathrm{P}>0.05)$, and hospitalization period $(\mathrm{P}>0.05)$ were not significantly different in the first and second weeks of the study between patients who died and the recovered or survived individuals (Tables 1 and 2). However, it was found that the mean of sodium level was $146.26 \mathrm{mEq} / \mathrm{L}$ in patients who died and 142.66 in alive patients $(\mathrm{P}<0.001)$ in the first week and 147.8 in the patients who died and 144.03 in alive patients $(\mathrm{P}<0.001)$ in the second week (Table 2). According to paired-samples T-test, the changes of sodium, potassium, mean arterial pressure, heart rate, GCS, calcium, phosphorous, magnesium, PT, PTT, and INR in the second week were significantly different from the parameter changes in the first week $(\mathrm{P}<0.05)$.

It was also demonstrated that 19 patients (19\%) in the first week and 15 patients (15\%) in the second week were 


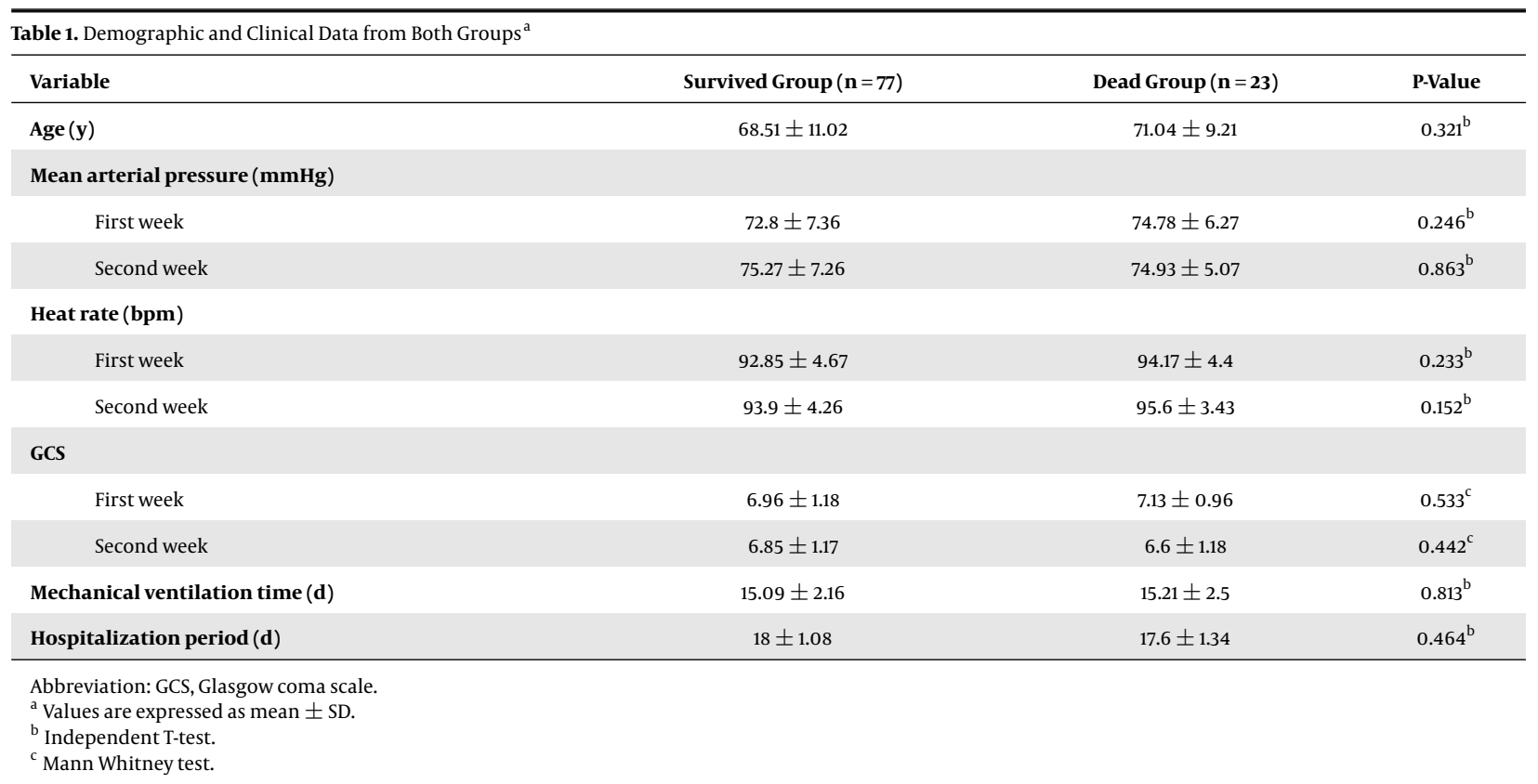

diagnosed with hypernatremia, and 17 (17\%) in the first week and seven patients (7\%) in the second week had hyponatremia. Our study results showed that out of 23 people who died, eight (34.78\%) died in the first week and 15 (65.21\%) died in the second week. Of the 23 patients who died, 14 (60.86\%) had hypernatremia, 5 (21.7\%) had hyponatremia, and four (17.39\%) had no electrolytic disorders (P $=0.037)$. It was also determined that out of 19 patients with hypernatremia in the first week, 6 (31.57\%) died, and eight (53.33\%) out of 15 patients who had hypernatremia in the second week died. It was also found that of 17 patients who had hyponatremia in the first week, 3 (17.64\%) died and two (28.57\%) out of seven patients who had hyponatremia in the second week died. In other words, in the first week, from eight cases who died, four patients (50\%) had hypernatremia, two patients (25\%) had hyponatremia, and two patients (25\%) were without electrolyte disorders $(\mathrm{P}=0.04)$. However, in the second week, of 15 deaths, 10 patients (66.66\%) had hypernatremia, three patients (20\%) had hyponatremia, and two patients (13.33\%) were without electrolyte impairment $(\mathrm{P}=0.029)$.

\section{Discussion}

The results of our study demonstrated that both hypernatremia and hyponatremia significantly increased mortality in TBI patients admitted to the ICU, but the total number of patients died from hypernatremia was significantly higher than that of hyponatremia. Despite the care that is being taken at special centers, electrolyte and acid-base disorders are usual. Generally, the cause of these disorders is the anaerobic metabolism caused by tissue hypoperfusion, and usually, the cause of this hypo-perfusion is a process called "autonomic storm", the activation of the autonomic system; On the other hand, changes that occur in the brain can lead to disorders such as diabetes insipidus, which ultimately leads to high urinary volume and reduced intravascular volume (9).

In a study conducted by Amini et al. (10), it was demonstrated that out of $150 \mathrm{TBI}$ patients, 34 individuals suffered from sodium deficiency. Hypernatremia was seen in $22 \mathrm{pa}-$ tients (19 males and 3 females) with mortality of 81.8\% (18 individuals). There was a significant difference in the mortality due to hypernatremia $(\mathrm{P}<0.001)$. In patients with hyponatremia, nine patients (all male) developed the syndrome of inappropriate antidiuretic hormone secretion, and three patients developed cerebral salt wasting. Three patients died. In this study, a significant relationship was seen between mortality and hypernatremia. The results of the study are in line with our findings. In a study by Waikar et al. (11), the investigators reported that even mild hyponatremia was independently associated with a higher risk of mortality in hospital and after a long period after discharge.

Another study by Mokhtari et al. (12), revealed that mortality rates were significantly higher in patients with hyponatremia (34\% vs. $16 \%$ ) and hypernatremia (55\% vs. $18 \%$ ) than in other patients. The mortality rate was signifi- 


\begin{tabular}{|c|c|c|c|}
\hline Variable & Survived Group & Dead Group & P-Value $^{\text {b }}$ \\
\hline \multicolumn{4}{|l|}{ Sodium (meq/L) } \\
\hline First week & $142.66 \pm 2.4$ & $146.26 \pm 5.24$ & $<0.001$ \\
\hline Second week & $144.03 \pm 2.35$ & $147.8 \pm 4$ & $<0.001$ \\
\hline \multicolumn{4}{|l|}{ Potassium (meq/L) } \\
\hline First week & $3.41 \pm 0.22$ & $3.42 \pm 0.4$ & 0.926 \\
\hline Second week & $3.46 \pm 0.24$ & $3.34 \pm 0.2$ & 0.064 \\
\hline \multicolumn{4}{|l|}{ Calcium (mg/dl) } \\
\hline First week & $8.68 \pm 0.28$ & $8.74 \pm 0.21$ & 0.397 \\
\hline Second week & $8.51 \pm 0.3$ & $8.4 \pm 0.3$ & 0.236 \\
\hline \multicolumn{4}{|l|}{ Phosphorus (mg/dl) } \\
\hline First week & $2.61 \pm 0.16$ & $2.64 \pm 0.12$ & 0.341 \\
\hline Second week & $2.55 \pm 014$ & $2.62 \pm 0.14$ & 0.122 \\
\hline \multicolumn{4}{|l|}{ Magnesium (mg/dl) } \\
\hline First week & $1.89 \pm 0.25$ & $1.92 \pm 0.23$ & 0.702 \\
\hline Second week & $1.79 \pm 0.21$ & $1.87 \pm 0.22$ & 0.22 \\
\hline \multicolumn{4}{|l|}{ PT (Second) } \\
\hline First week & $13.43 \pm 1.72$ & $13.36 \pm 1.02$ & 0.871 \\
\hline Second week & $13.72 \pm 2.21$ & $13.56 \pm 1.24$ & 0.785 \\
\hline \multicolumn{4}{|l|}{ PTT (Second) } \\
\hline First week & $22.53 \pm 1.89$ & $22.17 \pm 0.38$ & 0.371 \\
\hline Second week & $22.85 \pm 2.05$ & $23.06 \pm 2.12$ & 0.72 \\
\hline \multicolumn{4}{|l|}{ INR } \\
\hline First week & $1.42 \pm 0.43$ & $1.41 \pm 0.29$ & 0.962 \\
\hline Second week & $1.55 \pm 0.52$ & $1.53 \pm 0.37$ & 0.904 \\
\hline
\end{tabular}

${ }^{\text {a }}$ Values are expressed as mean $\pm \mathrm{SD}$.

${ }^{\mathrm{b}}$ Mann Whitney Test.

cantly higher in the hypernatremia group than in the hyponatremia group. At the end of the study, it was concluded that sodium disorders are common in ICU patients. These disorders are associated with increased mortality and are more common at elder ages; moreover, mortality rates are higher in hypernatremia. The results of this study are similar to those of our study.

Studies have shown that hypernatremia is associated with higher mortality and morbidity in general and surgical ICUs $(13,14)$. Hypernatremia is associated with an increased risk of mortality between $40 \%$ and $60 \%$ (13), which is approximately close to the mortality rate in the present study. Aiyagari et al. (15), showed that although mortality rate in patients admitted to the neurosurgery ICU increased with hypernatremia (30.1\% vs. $10.2 \%)$, only severe hypernatremia independently increased mortality in the neurosurgery ICU patients. One study has shown that the mortality rate in patients with hypernatremia is 39\% at the time of admission and 43\% during hospitalization, which is significantly higher than in patients without hypernatremia (24\% and $13 \%$ ). Other studies have also asserted the role of hypernatremia as an independent predictor of mortality. Lindner et al. (16) showed that hypernatremia is an independent risk factor for prediction of mortality in the general ICU (odds ratio 2/1).

On the other hand, acute hyponatremia can lead to death if brain edema is not treated promptly. Conversely, if chronic hyponatremia is rapidly corrected, osmotic demyelination occurs, which is potentially fatal. Typically, hyponatremia is a predictor of mortality in patients with advanced heart failure and liver cirrhosis. In these circumstances, it is generally assumed that hyponatremia is an 
indicator of the severity of the underlying disease rather than being directly involved in mortality. Considering the same subject, recent reports have mentioned the association between hyponatremia and mortality in patients with pulmonary embolism, pulmonary hypertension, pneumonia, and myocardial infarction. Recent data show that chronic mild hyponatremia is associated with mortality in the general population (17).

\subsection{Conclusion}

According to the results of this study, the prevalence of mortality is high in ICU patients who have sodium disorders. In case of improper treatment, these disorders might increase mortality remarkably. Mortality of hypernatremia is significantly higher than that of hyponatremia. Therefore, physicians need to consider carefully the risk factors, patients at risk, symptoms, prevention strategies, and treatment of these two disorders, especially hypernatremia. The limitation of this study was the small sample size and the fact that we followed patients with TBI for only two weeks; however, the findings seem particularly robust in spite of these limitations. In addition, we suggest altering the study design and further investigation.

\section{Acknowledgments}

This research is based on a professional medical doctoral thesis conducted under the spiritual and financial support of the research deputy of Isfahan University of Medical Sciences. In this way, we appreciate the efforts of these dear individuals.

\section{References}

1. Robertson GL. Diabetes insipidus. Endocrinol Metab Clin North Am. 1995;24(3):549-72. [PubMed: 8575409].

2. Doczi T, Bende J, Huszka E, Kiss J. Syndrome of inappropriate secretion of antidiuretic hormone after subarachnoid hemorrhage. Neurosurgery. 1981;9(4):394-7. [PubMed: 7301084].

3. Bradshaw K, Smith M. Disorders of sodium balance after brain injury. Cont Educ In Anaesth Crit Care Pa. 2008;8(4):129-33. doi: 10.1093/bjaceaccp/mkno19.
4. Gabbe BJ, Lyons RA, Lecky FE, Bouamra O, Woodford M, Coats TJ, et al. Comparison of mortality following hospitalisation for isolated head injury in England and Wales, and Victoria, Australia. PLoS One. 2011;6(5). e20545. doi: 10.1371/journal.pone.0020545. [PubMed: 21655237]. [PubMed Central: PMC3105093].

5. Levi M, van der Poll T, ten Cate H, van Deventer SJ. The cytokinemediated imbalance between coagulant and anticoagulant mechanisms in sepsis and endotoxaemia. Eur J Clin Invest. 1997;27(1):3-9. [PubMed: 9041370].

6. Maggiore U, Picetti E, Antonucci E, Parenti E, Regolisti G, Mergoni $\mathrm{M}$, et al. The relation between the incidence of hypernatremia and mortality in patients with severe traumatic brain injury. Crit Care. 2009;13(4):R110. doi: 10.1186/cc7953. [PubMed: 19583864]. [PubMed Central: PMC2750153].

7. Lisman T, Leuvenink HG, Porte RJ, Ploeg RJ. Activation of hemostasis in brain dead organ donors: an observational study. J Thromb Haemost. 2011;9(10):1959-65. doi: 10.1111/j.1538-7836.2011.04442.x. [PubMed: 21762465].

8. Barklin A. Systemic inflammation in the brain-dead organ donor. Acta Anaesthesiol Scand. 2009;53(4):425-35. doi: 10.111//j.13996576.2008.01879.x. [PubMed: 19226294].

9. Belzberg H, Shoemaker WC, Wo CC, Nicholls TP, Dang AB, Zelman V, et al. Hemodynamic and oxygen transport patterns after head trauma and brain death: implications for management of the organ donor. JTrauma. 2007;63(5):1032-42. doi:10.1097/01.ta.0000235995.86162.d2. [PubMed: 17993948].

10. Amini S, Heydari Z. [Sodium disturbances and its relation with mortality and morbidity in head trauma patients admitted to general ICU at Zahedan Khatam-Al-Anbia Hospital from May 2003 to September 2004]. J Iranian Soc Anaesthesiol And Intens Care. 2007;29(57):24-30. Persian.

11. Waikar SS, Mount DB, Curhan GC. Mortality after hospitalization with mild, moderate, and severe hyponatremia.Am JMed.2009;122(9):85765. doi: 10.1016/j.amjmed.2009.01.027. [PubMed: 19699382]. [PubMed Central: PMC3033702].

12. Mokhtari M, Goharani R, Miri M, Eghtesadi Araghi P. [Frequency of Hyper-and Hypo-natremia in Patients admitted in the ICU and comparison of their association with mortality]. Res Med.2010;33(3):183-8. Persian.

13. Palevsky PM. Hypernatremia. Semin Nephrol. 1998;18(1):20-30. [PubMed: 9459286].

14. Polderman KH, Schreuder WO, Strack van Schijndel RJ, Thijs LG. Hypernatremia in the intensive care unit: an indicator of quality of care? Crit Care Med.1999;27(6):1105-8. [PubMed:10397213].

15. Aiyagari V, Deibert E, Diringer MN. Hypernatremia in the neurologic intensive care unit: how high is too high? J Crit Care. 2006;21(2):16372. doi: 10.1016/j.jcrc.2005.10.002. [PubMed:16769461].

16. Lindner G, Funk GC, Schwarz C, Kneidinger N, Kaider A, Schneeweiss $B$, et al. Hypernatremia in the critically ill is an independent risk factor for mortality. Am J Kidney Dis. 2007;50(6):952-7. doi: 10.1053/j.ajkd.2007.08.016. [PubMed: 18037096].

17. Hoorn EJ, Zietse R. Hyponatremia and mortality: moving beyond associations. Am J Kidney Dis. 2013;62(1):139-49. doi: 10.1053/j.ajkd.2012.09.019. [PubMed: 23291150]. 